\title{
Instrumento para medir el desarrollo económico a través del empoderamiento del sector de bajos recursos
}

\section{Instrument to measure economic development through empowerment of the low-income sector}

\author{
Acosta Mellado Erika Ivett ${ }^{1}$, Ruíz Pérez Roberto ${ }^{2}$, Cejudo Galindo Eduardo ${ }^{3}$ \\ 1Allende \#1016, Cd. Obregón, Son., 6441090978, erika.acosta@itson.edu.mx, Instituto \\ Tecnológico de Sonora, Dirección de Ciencias Económico Administrativas, \\ Departamento de Contaduría y Finanzas/ ORC ID: 0000-0003-3526-8923 \\ ${ }^{2}$ Tamaulipas \#43, Esperanza, Son., 6441271374, roberto.ruiz@itson.edu.mx, Instituto \\ Tecnológico de Sonora, Dirección de Ciencias Económico Administrativas, \\ Departamento de Contaduría y Finanzas/ ORC ID: 0000-0001-8884-9890 \\ ${ }^{3}$ C Mar de Cortez \#1111, Cd. Obregón, Son., 6441389987, \\ eduardogalindo98@ hotmail.com
}

\section{DOI https://doi.org/10.46589/rdiasf.vi36.397}

Recibido 26 de junio 2021.

Aceptado 23 de septiembre 2021

Publicado 24 de octubre 2021

\section{Resumen}

Con el paso del tiempo la fortuna o el poder adquisitivo de las personas se ha divido, debido a las diferentes oportunidades que se presentan en lo largo de la vida, para unos pueden ser más favorables mientras que para otros son escazas o nulas. Se han tratado de implementar distintas propuestas para la integración del sector de bajos recursos a la cadena de valor. Esta investigación tiene como objetivo, Diseñar y validar un instrumento de medición para encontrar algunos factores críticos que coadyuven al logro de una integración exitosa del sector de bajos recursos en la cadena de valor. El instrumento es una adaptación al español del autor Dr. Mario César Dávila Aguirre, el proceso de validación de contenido se llevo a cabo por expertos. Como resultado en la validación del intrumento se obtuvo que hay ciertas preguntas que requieren ser modificadas para que el grado de validez aumente.

Palabras clave: Validación, Instrumento, Desarrollo Económico, Empoderamiento 


\section{Abstract}

With the passage of time, people's fortune or purchasing power has been divided, due to the different opportunities that arise throughout life, for some they may be more favorable while for others they are scarce or nil. They have tried to implement different proposals for the integration of the low-income sector into the value chain. This research aims to design and validate a measurement instrument to find some critical factors that contribute to the achievement of a successful integration of the low-income sector in the value chain. The instrument is an adaptation into Spanish by the author Dr. Mario César Dávila Aguirre, the content validation process was carried out by experts. As a result, in the validation of the instrument, it was obtained that there are certain questions that need to be modified so that the degree of validity increases.

Keywords: Validation, Instrument, Economic Development, Empowermen

\section{Introducción}

A pesar de ser un problema ancestral, el tema de la pobreza, ha resurgido con mayor énfasis en la agenda pública mundial en respuesta al objetivo número 1 propuesto por la Organización de las Naciones Unidas (ONU) en su Agenda 2030 sobre el Desarrollo Sostenible, el cual plantea poner fin a la pobreza en todas sus formas en todo el mundo (ONU, 2015). Además este mismo documento plantea, entre otras, dos de las principales formas de generar desarrollo: Promover la igualdad de género y empoderamiento de la mujer y; El trabajo decente y crecimiento económico.

Este objetivo también ha sido adoptado por otras organizaciones internacionales, como el Fondo Monetario Internacional, el Banco Mundial, el Banco Interamericano de Desarrollo, y la Organización para la Cooperación y el Desarrollo; sin embargo, el reto es enorme. En la actualidad, dos de cada tres personas en el mundo viven en la pobreza; la mayoría de ellos viven en las economías emergentes (Prahalad, 2004).

Esta parte de la población mundial ha sido llamada la "Base de la Pirámide", lo que indica un enorme tamaño de este grupo y la posición más baja en el orden económico (Prahalad \& Hart, 2002). Prahalad (2004) en su libro The Fortune at the Bottom of the Pyramid, señaló que más de 4 mil millones de personas viven con menos de 2 dólares estadounidenses por día. De acuerdo con Forbes (2017) México, ha 
alcanzado una población de pobreza de aproximadamente 53 millones 418,151.

Para entender mejor estos 53 millones de personas en México, la Asociación Mexicana de Agencias de Inteligencia de Mercado y Opinión (AMAI) agrupa y clasifica a los hogares mexicanos en siete niveles, de acuerdo a su capacidad para satisfacer las necesidades de sus integrantes.

El desarrollo del modelo de estimación del Índice de Niveles Socioeconómicos (NSE) está basado en un marco conceptual que considera seis dimensiones del bienestar dentro del hogar. La satisfacción de estas dimensiones determina la calidad de vida y bienestar de los integrantes de los hogares: 1) Capital Humano; 2) Infraestructura Práctica; 3) Conectividad y entretenimiento; 4) Infraestructura Sanitaria; 5) Planeación y futuro; 6) Infraestructura básica y espacio.

Actualmente la AMAI clasifica a los hogares utilizando la "Regla de NSE 2018”. Esta regla es un algoritmo desarrollado por el comité de Niveles Socioeconómicos que mide el nivel de satisfacción de las necesidades más importantes del hogar y produce un índice que clasifica a los hogares en siete niveles, considerando las siguientes seis características del hogar: 1) Escolaridad del jefe del hogar; 2) Número de dormitorios; 3) Numero de baños completos; 4) Número de personas ocupadas de 14 años y más; 5) Número de autos; y 6) Tenencia de internet. La Tabla 1 muestra la distribución de los hogares del país según Nivel Socioeconómico:

Tabla 1. Distribución de los hogares del país según Nivel Socioeconómico

\begin{tabular}{|c|c|}
\hline NACIONAL & \% \\
\hline $\mathbf{A} / \mathbf{B}$ & $6 \%$ \\
\hline $\mathbf{C}+$ & $11 \%$ \\
\hline $\mathbf{C}$ & $13 \%$ \\
\hline $\mathbf{C}-$ & $14 \%$ \\
\hline $\mathbf{D +}$ & $15 \%$ \\
\hline $\mathbf{D}$ & $30 \%$ \\
\hline E & $11 \%$ \\
\hline TOTAL & $100 \%$ \\
\hline
\end{tabular}

Fuente: Cálculos propios de la AMAI a partir de los datos de la Encuesta Nacional de Ingresos y Gastos de los Hogares 2016. 
Para efectos de esta investigación se definirá el sector de bajos recursos considerando los niveles de AMAI: D+, D y E, los cuales representan el 56\% de hogares de la población mexicana.

Avanzando en el conocimiento de los conceptos de la pobreza, es necesario comprender las mejores formas de erradicarla, particularmente en México. Por ejemplo, durante los últimos años, varias perspectivas empresariales, tales como, la corriente Base of the Pyramid (BOP) (Base de la Pirámide) (Prahalad \& Hart, 2002), y enfoque de Negocios (Empresas) socialmente inclusivos (Karnani, 2006) entre otros, han contribuido a la solución de este problema (Bruton, Ahlstrom \& Obloj, 2008). Así mismo, como parte de este fenómeno, la importancia de la participación de las multinacionales (corporaciones), el gobierno, y otros grupos de interés (stakeholders) es crucial para explicar cómo se pueden generar soluciones en el sector de bajos recursos en asociación.

De acuerdo con Narayan (2005), los pobres son el recurso más importante en la lucha contra la pobreza. Tienen imaginación, valor, conocimiento, experiencia, y una motivación profunda para superar la pobreza. El estudio Voces de los Pobres (Narayan, Chambers, Shah, \& Petesc, 2000) expuso, los pobres no son diferentes a las otras clases sociales en su deseo de vivir en un mundo seguro y tener acceso a los ingresos para que puedan educar, vestir y albergar a sus hijos con dignidad. Lo que desean es pertenecer y participar en sus comunidades en pie de igualdad con los demás. Por encima de todo, ellos no quieren caridad; quieren una oportunidad económica que les de rendimientos justos por su trabajo.

La teoría la Base de la Pirámide trajo de vuelta la vieja idea de que la comercialización orientada a los pobres en los países en desarrollo es la mejor manera de desarrollar las economías emergentes, al tiempo que aumentan las ganancias corporativas (Drucker, 1958; Porter, 1996). En este sentido, el término "Base de la Pirámide" se hizo popular debido a la promesa de una fortuna que se hará mediante la venta a los pobres captado la atención inmediata no sólo en el ámbito académico, sino también entre los profesionales de negocios.

Además de los practicantes, un coro creciente de estudiosos (por ejemplo, Delios, 2010; Guthrie \& Durand, 2008; Hinings \& Greenwood, 2002) durante los 
últimos años ha argumentado que es crucial para las empresas pensar más allá de la rentabilidad económica tomando un papel más activo y amplio en la sociedad.

Sin embargo, con pocas excepciones, se ha demostrado que la supuesta fortuna en la Base de la Pirámide no existe (Karnani, 2007; SEKN, 2011). Varios investigadores han dicho que la verdadera solución para aliviar, o al menos reducir, el nivel de pobreza no es ver a los pobres como clientes; en cambio, es importante integrar a estas personas en la cadena de valor de las corporaciones multinacionales (Karnani, 2007). En la literatura se puede encontrar un número creciente de estudios de caso que confirman la integración del sector de bajos recursos en la cadena de valor (por ejemplo, Unilever, Heineken, Cargill, ADM, Nestlé).

Reconocer que los patrones de estilo occidental de desarrollo económico pueden no ocurrir en estos entornos de negocio aumenta el éxito de las iniciativas dirigidas a mercados del sector de bajos recursos. Estas estrategias incluyen el desarrollo de relaciones con socios no tradicionales, la co-invención de soluciones personalizadas, y la construcción o fomento de la capacidad local. Estas estrategias sugieren la importancia de que las empresas multinacionales desarrollen una capacidad global de compromiso social (London \& Hart, 2004).

En América Latina, un análisis de 33 iniciativas de la red académica SEKN, entre 2006 y 2009, mostró que las pequeñas y medianas empresas (PYME), así como organizaciones de la sociedad civil, son más ágiles y abiertas a la internalización de las innovaciones necesarias para llevar a cabo un negocio inclusivo, en particular en lo que se refiere al trabajo colaborativo. En este sentido, el grupo de investigadores de SEKN refuerza el papel de las PYME, así como de las organizaciones de la sociedad civil en la implementación de negocios inclusivos, haciendo hincapié en que el sector de bajos recursos puede unirse a la cadena de valor como proveedores de grandes corporaciones (SEKN, 2011).

$\mathrm{Si}$ se considera que tales iniciativas nacen junto con los negocios y tienen su origen en actividades empresariales dentro de la organización, una teoría que explica el surgimiento de estas actividades es la teoría del emprendimiento institucional.

El concepto de emprendimiento institucional se refiere a la actividad de aprovechamiento de los recursos para crear nuevas instituciones o para transformar los existentes (DiMaggio, 1988; Fligstein, 1997). El papel de la actividad individual y 
organizacional en el emprendimiento institucional se pone de relieve, a través de las posiciones de los actores y la conectividad relacional entre ellos (Hargrave \& Van de Ven, 2006).

Los estudiosos del emprendimiento institucional se basan en la teoría institucional y examinan cómo las organizaciones influyen en el establecimiento de prácticas y demandas ampliamente aplicadas, tales como reglas, normas y estándares institucionales (DiMaggio, 1988). En la terminología de esta literatura, los emprendedores institucionales son actores que "crean un nuevo sistema de significado que vincula el funcionamiento de conjuntos dispares de instituciones" (Garud Jain, \& Kumaraswamy, 2002, p. 196).

Cuando los emprendedores institucionales establecen nuevas instituciones, puede surgir una situación social fragmentada con una gama de prácticas, estructuras de autoridad y redes sociales en términos de competencia de mercado, (Peter, Hofstetter, \& Hoffman, 2011). Los recursos descritos en el emprendimiento institucional varían desde factores de entrada comúnmente accesibles, como el capital financiero o humano, hasta recursos altamente complejos, como el capital social.

Varios recursos utilizados por los emprendedores institucionales se han explicado hasta la fecha: el capital económico; (Howard-Grenville, Hoffman, \& Bhattacharya, 2007), profundos conocimientos técnicos ("know-how”) (Maguire Hardy \& Lawrence, 2004); habilidades políticas, sociales y analíticas (Fligstein, 1997; Garud, Jain, \& Kumaraswamy (2002); Perkmann \& Spicer, 2007); y el capital social de una organización con actores institucionales (Hamprecht, 2006; Howard-Grenville, et. al., 2007).

Peters, Hofstetter, \& Hoffmann, (2011) introducen el concepto de recursos clave que permite a los empresarios institucionales establecer iniciativas de sostenibilidad voluntarias y oponerse a las prácticas institucionales existentes y emergentes aplicadas en las cadenas de suministro (Misangyi Weaver, \& Elms, 2008). Estas iniciativas se refieren a la integración de partes interesadas externas, la integración multifuncional y la implementación de la cadena de suministro.

Es importante conocer el papel de estos recursos en la generación de desarrollo del sector de bajos recursos. En su trabajo sobre la pobreza, Sen (1985) argumentó que el bienestar económico de los pobres se entendía mejor a través de sus capacidades que, 
a través de conceptos económicos más tradicionales, como la elección y el cumplimiento de los deseos. Esto condujo al "marco de capacidades" para la evaluación del bienestar individual, un enfoque que se apartó de los enfoques de bienestar tradicionales que normalmente equiparan el bienestar con la opulencia o la utilidad (Nussbaum, 1988; Robeyns, 2003; Sen, 1999).

A medida que los pobres adquieren y desarrollan más capacidades, pueden aprovechar las oportunidades económicas y sociales como un medio de integración en la cadena de valor. Ansari, Munir, \& Gregg, (2012) llaman a estas "iniciativas sostenibles", y también respaldan que, para generar desarrollo, la pobreza debe considerarse no solo como una falta de recursos financieros, sino también como una falta de capacidad y capital social.

El enfoque de libertad y capacidad de Sen (Dreze \& Sen, 1989; Sen, 1985, 1999) ofrece una perspectiva completamente diferente sobre el desarrollo de las personas empobrecidas, una que hasta ahora ha estado ausente en la mayoría de los estudios de la Base de la Pirámide. El enfoque ha re-conceptualizado el desarrollo económico. Argumenta que los ingresos reales son una métrica analíticamente inadecuada para hacer comparaciones de bienestar y que los esfuerzos utilitarios para reducir el bienestar y el desarrollo económico a solo ingresos reales como medio de satisfacer las preferencias son igualmente inadecuados.

La literatura sobre la estrategia de la Base de la Pirámide sugiere que proporcionar empleo a los no calificados y generar un consumo general puede ser suficiente para "empoderar" a la Base de la Pirámide y que ésta salga de la pobreza. En contraste, el enfoque de capacidades sugiere que el ingreso o el consumo son los medios, y no los fines, para lograr lo que las personas realmente sean valoradas como individuos o grupos.

La creación de capital social es una actividad crucial para implementar un enfoque de capacidades y debe entenderse como una forma intermedia de capital intelectual que consiste en el conocimiento en grupos y redes de personas (Nahapiet \& Ghoshal, 1998). Además, Burt (1992), sostiene que el capital social se refiere a los recursos de conocimiento integrados en una red de relaciones que no se limitan necesariamente a los intercambios de conocimientos internos. Esto puede basarse principalmente entre los empleados, pero los vínculos con actores externos como 
clientes y proveedores, entre otros, no deben descartarse por irrelevantes (Yount, Subramaniam, \& Snell, 2004).

Las organizaciones de enlace son la clave para estabilizar los entornos turbulentos y desarrollar las bases institucionales necesarias para trabajar con los pobres (Brown, 1991). El trabajo de estas organizaciones generalmente cubre la brecha que existe entre los sectores formales/desarrollados de la economía con aquellos que son informales/ subdesarrollados (Márquez, Reffico \& Berger, 2010).

Algunos ejemplos de casos exitosos en México que involucran la integración del sector de bajos recursos en la cadena de valor son Sigma Alimentos, con su proyecto "Fomento Lechero", Danone con su proyecto "Margarita", Pepsico con su proyecto "Girasol" y Starbucks con su proyecto C.A.F.E.

Un importante constructo para comprender mejor cómo algunas de estas empresas multinacionales pueden crear desarrollo en comunidades con altos niveles de pobreza es el empoderamiento. La Agencia Suiza para el Desarrollo y la Cooperación (SDC) conceptualiza el empoderamiento como un proceso de emancipación en el que los desfavorecidos están facultados para ejercer sus derechos, obtener acceso a los recursos y participar activamente en el proceso de conformación de la sociedad y toma de decisiones. Por lo tanto, las actividades de la SDC están diseñadas para fortalecer a los pobres mediante el fortalecimiento de la autoconfianza y la capacidad de desarrollar posibles soluciones por sí mismas.

Según Luttrell, Quiroz \& Scrutton (2007), adoptar un enfoque multidimensional para el empoderamiento requiere que esto se defina en términos de capacidades individuales y acción colectiva para abordar las desigualdades que son las causas de la pobreza. Un enfoque en el empoderamiento enfatiza que la pobreza no solo tiene que ver con los bajos ingresos, sino que también se deriva de la exclusión social y la falta de acceso al poder, la voz y la seguridad. Bandura (1995), ha demostrado experimentalmente que, cuando las creencias sobre la autoeficacia se manipulan independientemente del desempeño y las condiciones externas, el desempeño futuro se ve afectado.

Por lo tanto, el sentido interno de eficacia de una persona juega un papel causal independiente. Al revisar la literatura psicológica, Bandura (1998: p.53) concluye: "Las creencias de la gente de que pueden producir los efectos deseados por sus acciones 
influyen en las elecciones que hacen, sus aspiraciones, el nivel de esfuerzo y la perseverancia, la resistencia a la adversidad, la vulnerabilidad al estrés y la depresión".

Para explicar el proceso de mejora del desempeño y el crecimiento económico a través del empoderamiento se establece que el emprendimiento social puede ayudar a describir las formas en que las empresas multinacionales pueden generar empoderamiento.

Entre las variables relevantes en el proceso de empoderamiento para generar desempeño se encuentran la confianza con el proveedor de servicios (empresa), la estrategia de afrontamiento y la aversión al riesgo. En este proceso, se utilizarán las fases para desarrollar marcos conceptuales propuestos por Jabareen (2009).

Como se señaló anteriormente, la propuesta de la base de la pirámide trajo de vuelta la vieja idea de que el marketing dirigido a los pobres en los países en desarrollo es la mejor manera de desarrollar economías emergentes mientras se incrementan las ganancias corporativas (Drucker, 1958; Porter, 1996). Con pocas excepciones, se ha demostrado que la supuesta fortuna en la base de la pirámide no existe (Karnani, 2007; SEKN, 2011).

Varios investigadores han afirmado que la verdadera solución para aliviar o al menos reducir el nivel de pobreza es no ver a los pobres como clientes. En cambio, es importante integrar a estas personas en la cadena de valor de las empresas multinacionales como una de las estrategias para generar valor en el sector de bajos recursos (Karnani, 2007).

Sin embargo, esta integración no es fácil. Una de las razones de esto es que los hogares pobres no pueden obtener capital de los bancos tradicionales porque no tienen garantías para asegurar préstamos y los bancos tradicionales no quieren asumir los riesgos y costos de otorgar préstamos pequeños sin garantía. Sin este capital, las personas empobrecidas no pueden superar el nivel de subsistencia, que es una debilidad devastadora del sistema bancario formal (Karnani, 2007). Algunas de estas personas intentan obtener el capital que necesitan y no pueden obtener de los bancos formales a través de micro financieros.

Los microfinancieros utilizan prácticas contractuales y formas organizativas innovadoras para reducir los riesgos y costos de otorgar préstamos, como los préstamos a grupos, en lugar de a una sola persona (Karnani, 2007). Sin embargo, se ha 
identificado que los micropréstamos son más beneficiosos para los prestatarios que viven por encima del umbral de pobreza que para los prestatarios que viven por debajo del umbral de pobreza (Hulme y Mosley, 1996).

Una explicación para esto son las altas tasas de interés que ofrecen estos micro financieros por pequeñas cantidades de dinero. Esto podría deberse a que los clientes con mayores ingresos están dispuestos a asumir más riesgos, mientras que los prestatarios de menores ingresos tienden a obtener préstamos conservadores para proteger su subsistencia y es menos probable que inviertan en nueva tecnología, capital fijo o la contratación de mano de obra (Karnani, 2007).

Es necesario garantizar la integración de las personas del sector de bajos recursos, y antes de que puedan formar parte de tales iniciativas, necesitan, en la mayoría de los casos, obtener apoyo financiero. En algunos casos, esto puede ser proporcionado por el sistema de microfinanzas, pero en otros casos, el gobierno o las empresas pueden proporcionar este apoyo financiero directamente. Los productores del sector de bajos recursos enfrentan obstáculos sustanciales para acceder tanto a los mercados de productos como a los mercados de factores.

Les resulta difícil acceder libremente a los mercados de factores debido a sus debilitantes limitaciones de recursos y la falta de activos reconocidos importantes (London, Anupindi \& Sheth 2010). También enfrentan barreras importantes para vender sus bienes y servicios en el mercado de productos. Por estas razones, el papel de las empresas en las integraciones del sector de bajos recursos es crucial porque, sin su apoyo, los productores pueden ser víctimas fáciles de estas restricciones.

Las empresas pueden ayudar a los productores de a obtener acceso a los mercados de productos invirtiendo en adquisiciones y transporte y cotejando los requisitos de los clientes y comunicando estas especificaciones a los productores (London et al., 2010). Sin embargo, como podemos ver, uno de los factores más importantes para generar una integración exitosa del sector de bajos recursos a la cadena de valor como productores o proveedores es el involucramiento de las empresas en el proceso, como en el caso de los ejemplos antes mencionados Sigma Alimentos con su proyecto "Fomento Lechero", Danone con su proyecto "Margarita", Pepsico con su proyecto "Girasol", o Starbucks con su proyecto Café. 
Una de las claves del éxito es la generación de empoderamiento social y económico para generar las condiciones necesarias para iniciar las operaciones (con las creaciones de grupos de agricultores) y con la creación de financiamiento para obtener acceso a recursos financieros.

Pregunta de investigación

¿Es valido el diseño de un instrumento para medir el desarrollo economico, a través del empoderamiento: integración del sector de bajos recursos en la cadena de valor?

Objetivo

Diseñar y validar un instrumento de medición para encontrar algunos factores críticos que coadyuven al logro de una integración exitosa del sector de bajos recursos en la cadena de valor.

\section{Método}

Para la primera fase de la validación se utilizó un cuestionario con diversos apartados, adaptado al sector de los productores de leche. Siendo las variables a medir de esta investigación el empoderamiento, confianza, estrategia de afrontamiento y aversión al riesgo. Las preguntas para cada una fueron tomadas de la tesis doctoral del Dr. Mario César Dávila Aguirre (2013). En función a ello se estructuraron las dimensiones del instrumento resultando cuatro apartados: 1). Objetivo a manera de presentación ante el entrevistado; 2) Información del encuestado (incluye variables de control); 3). Preguntas relacionadas con las variables de control; 4). Descripción de la escala de evaluación de los ítems; 5) Preguntas relacionadas a las variables dependientes e independiente, al inicio se coloca una definición de las mismas.

Variables de control

Primero, como se ha dicho anteriormente, el objetivo para esta investigación es validar la inclusión de este sector de bajos recursos en la cadena de valor, por lo que en el apartado número 1 se incluyen medidas de niveles socioeconómicos. Para ello se utilizó el instrumento desarrollado por la Asociación Mexicana de Agencias de Investigación de Mercados y Opinión Pública (AMAI). Este instrumento clasifica a la población mexicana en seis segmentos o niveles socioeconómicos diferentes. El nivel socioeconómico en México se midió utilizando la regla 10x6 AMAI. Esta regla es un índice que clasifica a los hogares en siete niveles, teniendo en cuenta ocho 
características, incluidas las posesiones del hogar y el nivel de educación del jefe de hogar. También como variables de control, se pregunta al participante sus edades y géneros.

Variable independiente

Como variable independiente, fue seleccionado el desempeño de los productores de leche. En este caso, se seleccionaron dos sesgos de factores de cambio a través del tiempo: 1) Cambio en el número de vacas al inicio de las operaciones para cada agricultor y 2) Cambio en el número promedio de litros producidos por cada vaca.

Variables dependientes

Para efectos de esta investigación se eligió el empoderamiento psicológico en el sector de bajos recursos, una medida desarrollada por Spreitzer (1995). Esta escala utiliza cuatro dimensiones: significado, competencia, autodeterminación e impacto. Cada dimensión tiene tres elementos de medida para un total de doce elementos.

Los ítems de competencia se adaptaron de la escala de autoeficacia de Jones (1986). Los ítems de impacto fueron adaptados de la escala de indefensión de Ashforth (1989). Los elementos de significado se tomaron de Tymon (1988). Los ítems de autodeterminación fueron adaptados de la escala de autonomía de Hackman y Oldham (1980). Todas estas escalas se han utilizado en investigaciones anteriores $\mathrm{y}$ han producido estimaciones adecuadas de fiabilidad (Spreitzer, 1995).

Los ítems fueron anclados por una escala Likert de siete puntos $(1=$ completamente en desacuerdo a $7=$ completamente de acuerdo). Elementos de muestra: "Tengo confianza en mi capacidad para hacer mi trabajo" (competencia), "El trabajo que hago es muy importante para mí" (es decir), "Puedo decidir por mi cuenta cómo hacer mi trabajo" (autodeterminación) y "Mi impacto en lo que sucede en mi grupo es grande" (impacto).

Confiar se midió adaptando la escala de confianza entre organizaciones utilizada por Morgan y Hunt (1994) y también por Bansal, Irving \& Taylor (2004). Los ítems fueron anclados por una escala de siete puntos $(1=$ completamente en desacuerdo a $7=$ completamente de acuerdo). Elementos de muestra: "Siento que puedo confiar completamente en la empresa (Sigma Alimentos)" y "Mi proveedor de servicios (supervisor de Sigma Alimentos) me trata de manera justa y equitativa”. 
Para medir una estrategia de afrontamiento, se utilizó una escala desarrollada por Duhacheck (2005) para medir el apoyo instrumental de las parejas. Los ítems fueron anclados por una escala de siete puntos $(1=$ completamente en desacuerdo, a $7=$ completamente de acuerdo). Elementos de muestra: "Pregunte a amigos con experiencias similares qué lo hicieron "," Pídale a un amigo que me ayude a solucionar el problema (por ejemplo, cuando una vaca está enferma) ".

Y finalmente, para medir la aversión al riesgo, se usó la escala basada en el instrumento original desarrollado por Raju (1980), que posteriormente fue utilizada por Keaveney y Parthasarathy (2001) y Bao, Zhou \& Su (2003). Es unidimensional y solo se mide por tres elementos. Estos ítems también fueron anclados por una escala de siete puntos $(1=$ completamente en desacuerdo a 7 = completamente de acuerdo $)$. Elementos de muestra: "Soy cauteloso al probar nuevos procesos (por ejemplo, para mejorar la alimentación de las vacas)", "Nunca compro algo que no conozco a riesgo de cometer un error".

Las escalas originales para las variables se desarrollaron en idioma inglés. Para traducir las escalas al español con el fin de adaptarlas al contexto del sector de bajos recursos en Cd. Obregón, Son. México, se optó por utilizar un software de traducción de documentos técnicos.

Posterior al diseño del instrumento se eligieron los expertos que formarían parte del panel para la validación, se les hizo una invitación vía correo electrónico donde se explicó el objetivo, propósito de la misma, etc., así como también se adjuntó el enlace que da acceso a la videoconferencia, y su duración. También se les hizo saber a los expertos sobre los antecedentes de la construcción del instrumento.

Los participantes todos docentes e investigadores del Instituto Tecnológico de Sonora, con posgrado y antigüedad laboral de entre $\operatorname{los} 8$ a 25 años, sus edades se oscilan entre 40 a 50 años.

De los 7 que participaron, todos entregaron los resultados de la evaluación sobre los distintos factores que influyen para la integración del sector de bajos recursos en la cadena de valor. Acerca de la participación como juez experto, Garrote y Rojas (2015) hacen referencia a la relevancia de fungir como tal; indican que esa labor es 
fundamental para "eliminar aspectos irrelevantes, incorporar los que son necesarios o modificar aquellos que lo requieran".

A los expertos presentes, el día de la evaluación, se les compartió una plantilla (Ver anexo 1) que incluía los ítems, con los criterios a evaluar. Los cuestionarios, cuyos criterios evalúan cada uno de los indicadores, permiten estimar el grado validez del instrumento; principios básicos de calidad que debe reunir tras ser visto y revisado por juicio de expertos (Garrote y Rojas, 2015. Los resultados derivados se presentan después del análisis estadístico.

Para ello se establecieron cuatro criterios, con las siguientes opciones de respuesta:

1. No cumple con el criterio; 2. Bajo nivel; 3. Moderado nivel; 4. Alto nivel

Criterios a evaluar:

- Suficiencia: Los ítems que pertenecen a una misma dimensión bastan para obtener la medición de ésta.

- Claridad: El ítem se comprende fácilmente, es decir, su sintáctica y semántica son adecuadas.

- Coherencia: El ítem tiene relación lógica con la dimensión o indicador que está midiendo.

- Relevancia: El ítem es esencial o importante, es decir debe ser incluido.

| Ya con la información adquirida, se realizó el análisis estadístico, para definir el grado en que los jueces están de acuerdo. Después de eso se aplicó el coeficiente de concordancia W de Kendall. Se aplica el coeficiente de concordancia de W Kendall, para medir el grado de correlación y consistencia interna de las variables que existe entre los expertos.

De acuerdo con (Siegel \& Castellan, 1995) citado por Escobar y Cuervo (2008) este coeficiente se utiliza cuando se quiere conocer el grado de asociación entre $\mathrm{k}$ conjuntos de rangos, el más común por ejemplo de 1 a 4 . El mínimo valor asumido por el coeficiente es 0 y el máximo 1. En cambio, hay que hacer la excepción que, hay que revisar la calificación dada a cada ítem, ya que puede haber una alta concordancia en los aspectos, un ejemplo de ello es que el ítem no sea adecuado. 


\section{Resultados}

Las tablas siguientes son de elaboración propia haciendo uso los resultados obtenidos por los expertos. Su estructura se divide de la siguiente manera, primero se presenta el Grado de concordancia, de cada una de las variables consideradas para el instrumento de medición, obtenido según el coeficiente $\mathrm{W}$ de Kendall (tomando en cuenta el parámetro menor que $(<)$ 1), después los criterios, suficiencia, claridad, coherencia y relevancia en el mismo orden de presentación de las variables considerando un rango de 1 a 4 para definir el grado de validez de cada uno.

Los resultados obtenidos, mostrados en la Tabla 2, en cuanto al grado de concordancia externa asignado por los expertos para cada uno de los ítems sobre la variable de Empoderamiento, indican que el nivel es significativo entre los rangos, pues se observa que ninguno excede el valor crítico de 0.1 , por lo que se estima que hay concordancia entre los jueces y muestra que los indicadores son adecuados, aunque susceptibles de mejora, ya que el análisis determina relaciones significativas entre criterios e indicadores.

Tabla 2. Resultados de consistencia externa de W Kendall en Empoderamiento

\begin{tabular}{ccc}
\hline Ítem & W de Kendall & $\boldsymbol{P}$ \\
\hline 1 & 0.857 & $\mathrm{p}<1$ \\
2 & 0.714 & $\mathrm{p}<1$ \\
3 & 0.714 & $\mathrm{p}<1$ \\
4 & 0.571 & $\mathrm{p}<1$ \\
5 & 0.571 & $\mathrm{p}<1$ \\
6 & 0.714 & $\mathrm{p}<1$ \\
7 & 0.714 & $\mathrm{p}<1$ \\
8 & 0.714 & $\mathrm{p}<1$ \\
9 & 0.571 & $\mathrm{p}<1$ \\
10 & 0.257 & $\mathrm{p}<1$ \\
11 & 0.095 & $\mathrm{p}<1$ \\
12 & 0.184 & $\mathrm{p}<1$ \\
\hline
\end{tabular}

Fuente. Elaboración propia, 2020. 
No hubo ningún ítem que contara con un nivel de concordancia absoluta, pero el más cercano a uno fue el ítem 1, éste se refiere a si el trabajo que hace es importante para el productor con un valor de 0.857 , mientras que el nivel más bajo fue el ítem 11 , que se refiere a si el productor tiene un gran control y conocimiento sobre lo que pasa dentro del grupo, con un nivel 0.095, esto nos indica que el ítem se puede fortalecer o simplemente quitarlo para que el grado de validez del instrumento sea aún mayor.

En la Tabla 3, se observan las evaluaciones obtenidas por los expertos sobre cada ítem. El ítem que tuvo más alta puntuación fue el 9, mientras que para la puntuación más baja hubo un empate con el número 2 y 3 . Analizando detenidamente la tabla no hubo ningún ítem que tuviera un nivel absoluto en los criterios, por lo que se pueden modificar para fortalecer la validación de la misma.

Tabla 3. Análisis cuantitativo de evaluaciones por criterio de los ítems de Empoderamiento

\begin{tabular}{ccccc}
\hline Ítem & Suficiencia & Claridad & Coherencia & Relevancia \\
\hline 1 & 3.57142857 & 3 & 3.42857143 & 3.57142857 \\
2 & 3.28571429 & 3 & 3.28571429 & 3.28571429 \\
3 & 3.28571429 & 3 & 3.28571429 & 3.28571429 \\
4 & 3.71428571 & 3.285714 & 3.42857143 & 3.71428571 \\
5 & 3.71428571 & 3.285714 & 3.57142857 & 3.57142857 \\
6 & 3.71428571 & 3.285714 & 3.57142857 & 3.57142857 \\
7 & 3.57142857 & 3.285714 & 3.57142857 & 3.57142857 \\
8 & 3.57142857 & 2.571429 & 3.57142857 & 3.42857143 \\
9 & 3.71428571 & 3.428571 & 3.71428571 & 3.57142857 \\
10 & 3.57142857 & 3 & 3.71428571 & 3.71428571 \\
11 & 3.42857143 & 3 & 3.57142857 & 3.57142857 \\
12 & 3.71428571 & 3 & 3.57142857 & 3.71428571 \\
\hline
\end{tabular}

Fuente. Elaboración propia, 2020 
En la Tabla 4 los resultados obtenidos en cuanto al grado de concordancia externa asignado por los expertos para cada uno de los ítems sobre la variable de Confianza, en este caso no se presentaron ítems que contaran con un nivel de concordancia absoluta, los más cercanos a 1 fueron los ítems 7 y 8 , el primero se refiere a si mi proveedor de servicio me trata con confianza y justicia y el segundo a, siento que puedo contar con mi proveedor de servicio cuando necesito su ayuda ambos con un nivel de 0.381 , mientras que el nivel más bajo fue el ítem 9, que se refiere a si el productor siento que mi proveedor de servicio no me toma en cuenta lo suficiente con un nivel de 0.02 , esto nos indica que el ítem se puede fortalecer o simplemente quitarlo para que el grado de validez del instrumento sea aún mayor.

Tabla 4. Resultado de consistencia externa de W de Kendall en Confianza

\begin{tabular}{ccc}
\hline Ítem & W de Kendall & $\boldsymbol{P}$ \\
\hline 1 & 0.095 & $\mathrm{p}<1$ \\
2 & 0.095 & $\mathrm{p}<1$ \\
3 & 0.095 & $\mathrm{p}<1$ \\
4 & 0.02 & $\mathrm{p}<1$ \\
5 & 0.184 & $\mathrm{p}<1$ \\
6 & 0.184 & $\mathrm{p}<1$ \\
7 & 0.381 & $\mathrm{p}<1$ \\
8 & 0.381 & $\mathrm{p}<1$ \\
9 & 0.02 & $\mathrm{p}<1$ \\
\hline
\end{tabular}

Fuente. Elaboración propia, 2020

La Tabla 5 revela que el ítem con la más alta puntuación fue el 8, alcanzando un nivel más alto sobre los otros en los cuatro criterios con puntuación de 4 en cada uno, mientras que la puntuación más baja fue para el ítem 4. Comparando los resultados de la tabla solo el ítem 8 tuvo un nivel absoluto en los criterios, por lo que se pueden modificar o agregar los demás para fortalecer la validación de la misma. 
Tabla 5. Análisis cuantitativo de evaluaciones por criterio de los ítems de Confianza

\begin{tabular}{crrrr}
\hline Ítem & Suficiencia & Claridad & Coherencia & Relevancia \\
\hline 1 & 3.57142857 & 3.285714 & 3.71428571 & 3.85714286 \\
2 & 3.71428571 & 3.571429 & 3.85714286 & 3.85714286 \\
3 & 3.57142857 & 3.714286 & 3.57142857 & 3.85714286 \\
4 & 3.42857143 & 3 & 3.28571429 & 3.57142857 \\
5 & 3.28571429 & 3.285714 & 3.57142857 & 3.71428571 \\
6 & 3.42857143 & 3.285714 & 3.42857143 & 3.71428571 \\
7 & 4 & 3.857143 & 3.85714286 & 4 \\
8 & 4 & 4 & 4 & 4 \\
9 & 3.85714286 & 3.857143 & 3.85714286 & 3.71428571 \\
\hline
\end{tabular}

Fuente. Elaboración propia, 2020.

La Tabla 6 devela que en la variable Aversión al riesgo, no hubo ningún ítem que contara con un nivel de concordancia absoluta, pero el más cercano a fue el ítem 1 y 2 , este se refiere soy muy cauteloso(a) al tratar de probar nuevas cosas y nunca compro algo si no conozco el riesgo que pueda tener con un nivel de 0.381 , mientras que el nivel más bajo fue el ítem 3, que se refiere a Usualmente compro cosas nuevas para poder probarlas con un nivel de 0.184 , esto nos indica que el ítem se puede fortalecer o simplemente quitarlo para que el grado de validez del instrumento sea aún mayor.

Tabla 6. Resultado de consistencia externa de W de Kendall en Aversión al riesgo

\begin{tabular}{ccc}
\hline Ítem & $\begin{array}{c}\text { W de } \\
\text { Kendall }\end{array}$ & $\boldsymbol{P}$ \\
\hline 1 & 0.381 & $\mathrm{p}<1$ \\
2 & 0.381 & $\mathrm{p}<1$ \\
3 & 0.184 & $\mathrm{p}<1$ \\
\hline
\end{tabular}

Fuente. Elaboración propia, 2020. 
Finalmente, la Tabla 7, muestra como el ítem 1, fue el que alcanzó un nivel más alto sobre los otros en los cuatro criterios, mientras que para la puntuación más baja fue el ítem 2. Examinando la tabla ningún ítem tuvo un nivel absoluto en los criterios, por lo que se pueden modificar o agregar los demás para fortalecer la validación de la misma.

Tabla 7. Análisis cuantitativo de evaluaciones por criterio de los ítems de Aversión al Riesgo

\begin{tabular}{ccccc}
\hline Ítem & Suficiencia & Claridad & Coherencia & Relevancia \\
\hline 1 & 3.71428571 & 3.428571 & 3.71428571 & 3.71428571 \\
2 & 3.57142857 & 3.571429 & 3.42857143 & 3.57142857 \\
3 & 3.5 & 3.666667 & 3.57142857 & 3.66666667 \\
\hline
\end{tabular}

Fuente. Elaboración propia, 2020

Comentarios de expertos

Así como los expertos contestaron el instrumento también hicieron comentarios de retroalimentación hacía el instrumento para el mejoramiento del mismo.

- En las variables de control:

La pregunta 3, Contando todos los focos que utilizan para iluminar su hogar, incluyendo los de techos, paredes y lámparas de buró o piso, dígame ¿cuántos focos tiene su vivienda? hubo un comentario respecto a esta, ¿Solo al interior? ¿En el exterior de la casa ¿patio frontal o patio trasero no se incluyen? Porque para mí el hogar, incluye vivienda y patio. Haciendo referencia a que se puede ser un poco más específico.

En la pregunta 4, se podría separar en 3 opciones de respuesta, pues que se englobe tierra y cemento no permitirá distinguir claramente el reactivo, pues es muy diferente que sea piso de tierra a piso de cemento, más si quieres identificar las personas de ingresos bajos del resto.

En la pregunta $6, ¿$ ¿En su hogar cuenta con estufa de gas o eléctrica? la respuesta es sí o no, se sugiere replantear la respuesta. 
- En la variable Empoderamiento:

En las preguntas del 1 al 3 ¿Qué diferencia hay entre: El trabajo que hago es muy importante para mí; Las actividades que hago tienen un significado muy importante para mí; ¿El trabajo que hago tiene un gran significado para mí? El experto comento: creo entender la diferencia, pero podría prestarse a confusión, entre los reactivos.

La pregunta 8, “¿Puedo tomar la decisión para saber cómo hacer mi trabajo?”, un experto comento que se puede modificar o replantear con el fin de seleccionar del 1 al 7. Misma retroalimentación para la pregunta 10 .

Para las preguntas 11 y 12 se hizo una observación en cuanto a la palabra que incluye "gran", ya que no se puede medir.

- Respecto a las peguntas de Desempeño, un experto comento: en las variables anteriores, las preguntas están relacionadas con la percepción personal de cada sujeto participante sobre sí mismo, en cambio los reactivos o indicadores de desempeño están orientados o enfocados al desempeño organizacional... ¿esto fue intencional? Los califiqué con 2 porque me parecen insuficientes, poco claros (al inicio de la empresa o de pertenecer a la empresa).

\section{Discusión}

El instrumento elaborado, con la intención de analizar y valorar los descriptores, y comprobar si los ítems miden las categorías que se desean medir en su coeficiente de concordancia externa, mediante coeficiente W de Kendall, muestra una concordancia significativa entre jueces, y pese a que algunos de los ítems manifiestan un alto índice de concordancia, también este procedimiento suministra información del grado de ambigüedad o debilidad de los constructos que presentaron bajo nivel de concordancia entre los expertos, lo que los hace factibles de revisión y mejoramiento.

Se enfatiza también que, parte fundamental del juicio de expertos es el nivel de experticia en la temática, pues se pretende que la opinión de los jueces sea informada y basada en su trayectoria en el tema (Escobar y Cuervo, 2008), y al ser ésta una problemática (empoderamiento del sector de bajos recursos) introducida recientemente en el ámbito académico y en contextos mexicanos, los expertos no cuentan con los antecedentes demandados en este tipo de estudios, por lo que los criterios de evaluación 
pudieran presentar cierto sesgo, determinado por la subjetividad de las respuestas de algunos de los jueces (Garrote y Rojas, 2015), que se manifiesta en la confiabilidad de los resultados obtenidos.

\section{Conclusión}

El proceso que se llevo a cabo fue con el objetivo de comprobar si el instrumento es valido para determinar factores que pueden influir en el desempeño de los productores de leche, esto mediante la evaluacion y juicio de un grupo de expertos. De esta manera, los resultados muestran el grado de validez que se obtuvo con la informacion recopilada por parte de lo expertos y también haciendo uso del coeficiente W de Kendall.

Una vez que el instrumento fue puesto bajo el juicio de los expertos para determinar validez en los criterios de suficiencia, claridad, coherencia y relevancia, en las variables de control, y las independientes empoderamiento, confianza y aversion al riesgo, se concluye que hay bastante concordancia entre los rangos asignadospor los jueces. Sin embargo, los resultados obligan a incrementar el indice de validez entre los expertos para que se vuelva más representativa y que cuente con mayor efectividad ypueda medir el desempeño de los productores en funcion del empoderamiento.

\section{Referencias bibliográficas}

Ansari, S., Munir, K. \& Gregg, T. (2012), Impact at the 'Bottom of the Pyramid': The Role of Social Capital in Capability Development and Community Empowerment. Journal of Management Studies. doi: 10.1111/j.14676486.2012.01042.

Ashforth, BE (1989). La experiencia de la impotencia en las organizaciones. Comportamiento organizacional y procesos de decisión humana, 43: 207-242.

Bandura, A. (1995). Comments on the Crusade against the Causal Efficacy of Human Thought. Journal of Behavior Therapy and Experimental Psychiatry 26: 17990.

Bandura, A. (1998). Personal and Collective Efficacy in Human Adaptation and Change. Advances in Psychological Science, vol. 1, Social, Personal, and Cultural Aspects, ed. J. G. Adair, D. Belanger, and K. L. Dion, 51-71. Hove, UK: Psychology Press. 
Bao Y, Zhou KZ, Su C. (2003). Enfrentar la conciencia y la aversión al riesgo: ¿afectan la toma de decisiones del consumidor? Marketing de psicología; 20 (8): 73355.

Bansal, HS, Irving, PG y Taylor, SF (2004). Un modelo de tres componentes de cliente a proveedores de servicios. Revista de la Academia de Ciencias del Marketing, $32(3), 234-250$.

Brown, L. D. (1991). Bridging Organizations and Sustainable Development. Human Relations, 44(8), 807-831.

Bruton, G. D., Ahlstrom, D., \& Obloj, K. (2008). Entrepreneurship in emerging economies: where are we today and where the research should go in the future. Entrepreneurship: Theory \& Practice, 32(1), 1-14.

Burt, R. (1992). Structural holes: The social structure of competition. Cambridge, MA: Harvard University Press.

Davila Aguirre Mario Cesar (2013). Desarrollo mediante empoderamiento: integración del sector de bajos ingresos en la cadena de valor.

Delios, A. (2010). How can organizations be competitive but dare to care? Academy of Management Perspectives, 24, 25-36.

DiMaggio, Paul (1988). Interest and Agency in Institutional Theory. Research on Institutional Patterns: Environment and Culture, pp. 3-21. Cambridge, MA: Ballinger Press.

Drèze, J., Sen, A., (1989). Hunger and Public Action. Clarendon Press, Oxford.

Drucker, P. F. (1958). Marketing and economic development. Journal of

Marketing, 22, 252-259.

Duhachek, A. (2005). Afrontamiento: un marco jerárquico multidimensional de respuestas a episodios de consumo estresante. Revista de investigación del consumidor, 32 (1), 41-53.

Escobar J., Cuervo Ángela. (2008). Validez de contenido y juicio de expertos: una aproximación a su utilización. Noviembre 16, 2020, de Universidad Nacional de Colombia

FORBES México. Recuperado de https://www.forbes.com.mx/mexico-tiene-534-millones-de-pobres/ 
Fligstein, N. (1997). Social skill and institutional theory. American Behavioral Scientist, 40: 397-405.

Garud, R., Jain, S., Kumaraswamy, A., (2002). Orchestrating institutional processes for technology sponsorship: the case of Sun Microsystems and Java. Academy of Management Journal 45, 196-214.

Garrote, P. R., \& del Carmen Rojas, M. (2015). La validación por juicio de expertos: dos investigaciones cualitativas en Lingüística aplicada. Revista Nebrija de lingüística aplicada a la enseñanza de lenguas, (18), 124-139.

Guthrie, D. \& Durand, R. (2008). Social issues in the study of management. European Management Review, 5, 137- 49.

Hackman, J. R., Hackman, R. J., \& Oldham, G. R. (1980). Work redesign (Vol. 2779). Reading, Mass.: Addison-Wesley.

Hamprecht, J. (2006), Sustainable Purchasing Strategy in the Food Industry, Difo, St Gallen.

Hargrave, T.J. and van de Ven, A.H. (2006), A collective action model of institutional innovation, Academy of Management Review, Vol. 31 No. 4, pp. 864-88

Hinings, C. and Greenwood, R. (2002). Disconnects and consequences in organization theory?. Administrative Science Quarterly, 47, 411-21.

Howard-Grenville, J.A., Hoffman, A.J. and Bhattacharya, C.B. (2007), Who can act on sustainability issues? Corporate capital and the configuration of organizational fields", in Sharma, S., Starik, M. and Husted, B. (Eds), Organizations and the Sustainability Mosaic: Crafting Long-Term Ecological and Societal Solutions, Edward Elgar Publishing, Cheltenham and Northampton, MA, pp. 193-21.

Hulme, D., \& Mosley, P. (1996). Finance against poverty (Vol. 2). Psychology Press.

Jabareen, Y. (2009). Building a conceptual framework: philosophy, definitions, and procedure. International journal of qualitative methods, 8(4), 49-62.

Jones, A. H. M. (1986). The later Roman Empire, 284-602: a social economic and administrative survey (Vol. 2). taylor \& Francis.

Karnani, A. (2006). Fortune at the bottom of the pyramid: a mirage. How the private sector can help alleviate poverty. Ross School of Business. Working Paper Series 1035 . 
Karnani, A. (2007). Microfinance misses its mark. Stanford Social Innovation revisión, 5 (3), 34-40.

Keaveney, S. y Parthasarathy, M. (2001), Comportamiento de cambio de cliente en los servicios en línea: un estudio exploratorio del papel de factores demográficos, conductuales y de actitud seleccionados. Revista de la Academia de Ciencias del Marketing, vol. 29, núm. 4, págs. 374-90.

London, T., Anupindi, R., \& Sheth, S. (2010). Creating mutual value: Lessons learned from ventures serving base of the pyramid producers. Journal of Business Research, 63(6), 582-594.

London, T., \& Hart, S. (2004). Reinventing strategies for emerging markets: beyond the transnational model. Journal of International Business Studies, 35(5), 350-370.

Luttrell, C., Quiroz, S., y Scrutton, C. (2007). Empowerment: An overview. Berne, Switzerland and Brighton, U.K.: Intercooperation (Swiss Foundation for Development and International Cooperation) and the Overseas Development Institute.

Maguire, S., Hardy, C., Lawrence, TB, (2004). Institutional entrepreneurship in emerging fields: HIV/AIDS treatment advocacy in Canada. Academy of Management Journal 47 (5), 657 - 679.

Márquez P., Reficcio E. and Berger G. (2010). Socially Inclusive Business engaging the poor through market initiatives in Iberoamerica, Social Enterprise Knowledge Network, Harvard, USA.

Misangyi, V.F., Weaver, G.R. and Elms, H. (2008), Ending corruption: the interplay among institutional logics, resources, and institutional entrepreneurs, Academy of Management Review, Vol. 33 No. 3, pp. 750-70.

Morgan, RM y Hunt, SD (1994). La teoría compromiso-confianza del marketing relacional. Revista de marketing, 58 (3), 20-38.

Nahapiet, J., y Ghoshal, S. (1998). Social capital, intellectual capital, and the organizational advantage. Academy of Management Review, 23(2): 242-266.

Narayan, D. (2005). Conceptual Framework and Methodological Challenges. In D. Narayan (Red.), Measuring Empowerment: CrossDisciplinary Perspectives (s. 3 38). Washington: The World Bank. 
Narayan, D., Chambers, R., Shah, MK, y Petesch, P. (2000). Voices of the Poor: Crying Out for Change. Bank New York (Vol. 2, p. book). Oxford University Press.

Nussbaum, M. (1988). Nature, function and capability: Aristotle on political distribution. Oxford Studies in Ancient Philosophy, Supplement, 145-84.

Organización de las Naciones Unidas (ONU), 2015. Recuperado de: https://www.un.org/sustainabledevelopment/es/

Perkmann, M. y Spicer, A. (2007), Healing the scars of history: projects, skills and field strategies in institutional entrepreneurship, Organization Studies, vol. 28 No. 7, pp. 1101- 22.

Peters, NJ, Hofstetter, JS, y Hoffmann, VH (2011). Institutional entrepreneurship capabilities for interorganizational sustainable supply chain strategies. The International Journal of Logistics Management, 22(1), 52-86.

Porter, ME (1996). What is strategy, Harvard Business Review Nov/Dec, 61-78.

Prahalad, CK (2004). The fortune at the bottom of the pyramid: eradicating poverty through profits. Wharton School Publishing.

Prahalad, CK, y Hart, S. (2002). The fortune at the bottom of the pyramid. Booz Allen Hamilton, strategy + business, 26 (enero).

Raju, PS (1980), Nivel de estimulación óptimo: su relación con la personalidad, la demografía y el comportamiento exploratorio, Journal of Consumer Research, 7 (diciembre), 272-282.

Robeyns, I. (2003). Sen's capability approach and gender inequality: selecting relevant capabilities. Economía Feminista, 9, 61-92.

SEKN (Enterprise Knowledge Network Social) (2011). Inclusive business: engaging the poor through market initiatives in Iberoamerica. Cambridge, MA: Centro David Rockefeller de Estudios Latinoamericanos, Harvard University Press.

Sen, A. (1985). A sociological approach to the measurement of poverty: a reply to Professor Peter Townsend. Oxford Economic Papers, 37(4), 669-676.

Sen, A. (1999). Development as Freedom Oxford: Oxford University Press.

Siegel, S., \& Castellan, N. J. (1995). Estadística no paramétrica: aplicada a las ciencias de la conducta (Vol. 4, pp. 195-196). México: Trillas.

Spreitzer, GM (1995). Psicológico, Empoderamiento en el Trabajo: Dimensiones, Medición y Validación. Academy of Management Journal, 38 (5), 1442-1465. 
Spreitzer, GM, Kizilos, MA y Nason, SW (1997). Un análisis dimensional de la relación entre empoderamiento psicológico y efectividad, satisfacción y tensión. Journal of Management, 23 (5), 679-704.

Tymon Jr, W. G. (1988). An empirical investigation of a cognitive model of empowerment. Doctoral diss., Temple University 1988. Dissertation Abstracts International, 49, 88-18.

Youndt, M., Subramaniam, M y Snell, S. (2004). Intellectual capital profiles: An examination of investments and returns. Journal of Management Studies, 41 (2), 335361.

\section{Como citar este artículo:}

Acosta Mellado, E., Ruíz Pérez, R. \& Cejudo Galindo, E. . (2021). Instrumento para medir el desarrollo económico a través del empoderamiento del sector de bajos recursos. Revista De Investigación Académica Sin Frontera: División De Ciencias Económicas $Y$ Sociales, (36). https://doi.org/10.46589/rdiasf.vi36.397

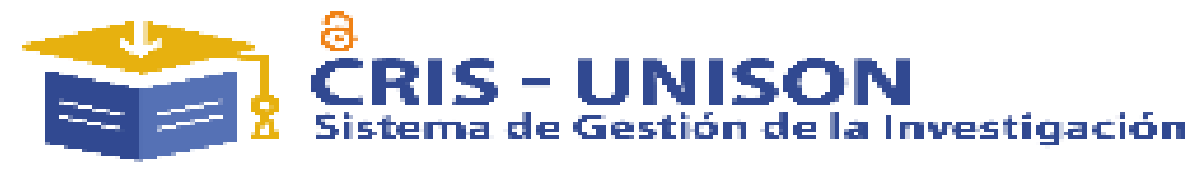

o Dialnet
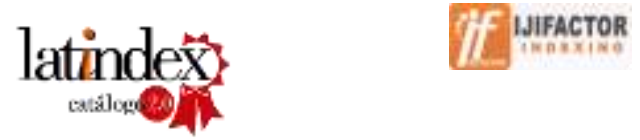

REDIB

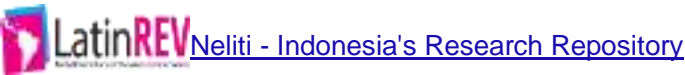
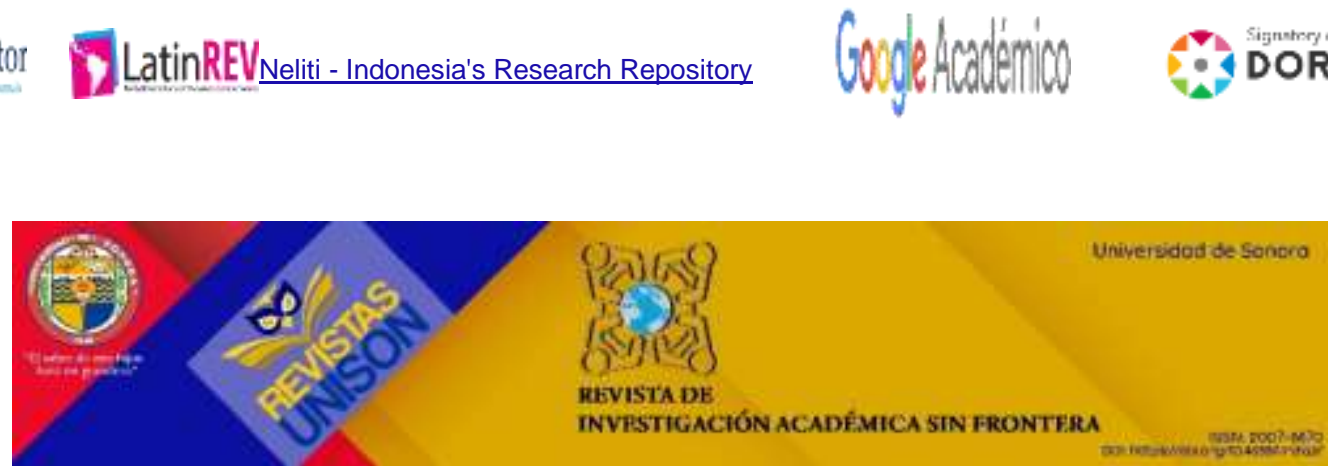


\section{Anexo 1}

\section{Cédula de evaluación}

1. No cumple con el criterio; 2. Bajo nivel; 3. Moderado nivel; 4. Alto nivel

Criterios a evaluar:

- Suficiencia: Los items que pertenecen a una misma dimensión bastan para obtener la medición de ésta.

- Claridad: El ítem se comprende fácilmente, es decir, su sintáctica y semántica son adecuadas.

- Coherencia: El ítem tiene relación lógica con la dimensión o indicador que está midiendo.

- Relevancia: El ítem es esencial o importante, es decir debe ser incluido.

\begin{tabular}{|c|c|c|c|c|}
\hline & $\begin{array}{l}\text { 1. No } \\
\text { cumple } \\
\text { con el } \\
\text { criterio; }\end{array}$ & $\begin{array}{c}\text { 2. Bajo } \\
\text { nivel }\end{array}$ & $\begin{array}{l}3 . \\
\text { Moderado } \\
\text { nivel }\end{array}$ & $\begin{array}{c}\text { 4. Alto } \\
\text { nivel }\end{array}$ \\
\hline Generales & Suficiencia & Claridad & Coherencia & Relevancia \\
\hline $\begin{array}{l}\text { ¿Cuál es el total de cuartos o } \\
\text { habitaciones con que cuenta su hogar? } \\
\text { Por favor no incluir baños, medios } \\
\text { baños, pasillos y patios. }\end{array}$ & & & & \\
\hline $\begin{array}{l}\text { ¿Su hogar cuenta con regadera } \\
\text { funcionado en alguno de los baños? }\end{array}$ & & & & \\
\hline $\begin{array}{l}\text { Contando todos los focos que utilizan } \\
\text { para iluminar su hogar, incluyendo los } \\
\text { de techos, paredes y lámparas de buró o } \\
\text { piso, dígame ¿cuántos focos tiene su } \\
\text { vivienda? }\end{array}$ & & & & \\
\hline $\begin{array}{l}\text { ¿El piso de su hogar es de tierra, o de } \\
\text { cemento, o de algún otro tipo de } \\
\text { acabado? }\end{array}$ & & & & \\
\hline $\begin{array}{l}\text { ¿Cuántos automóviles propios, tienen } \\
\text { en su hogar? }\end{array}$ & & & & \\
\hline $\begin{array}{l}\text { ¿En su hogar cuenta con estufa de gas o } \\
\text { eléctrica? }\end{array}$ & & & & \\
\hline $\begin{array}{l}\text { Pensando en la persona que aporta la } \\
\text { mayor parte del ingreso en su hogar, } \\
\text { ¿cuál fue el último grado de estudios } \\
\text { que completó? }\end{array}$ & & & & \\
\hline $\begin{array}{l}\text { ¿Cuántos baños completos con regadera } \\
\text { y WC (excusado) hay para uso } \\
\text { exclusivo de los integrantes de su } \\
\text { hogar? }\end{array}$ & & & & \\
\hline
\end{tabular}




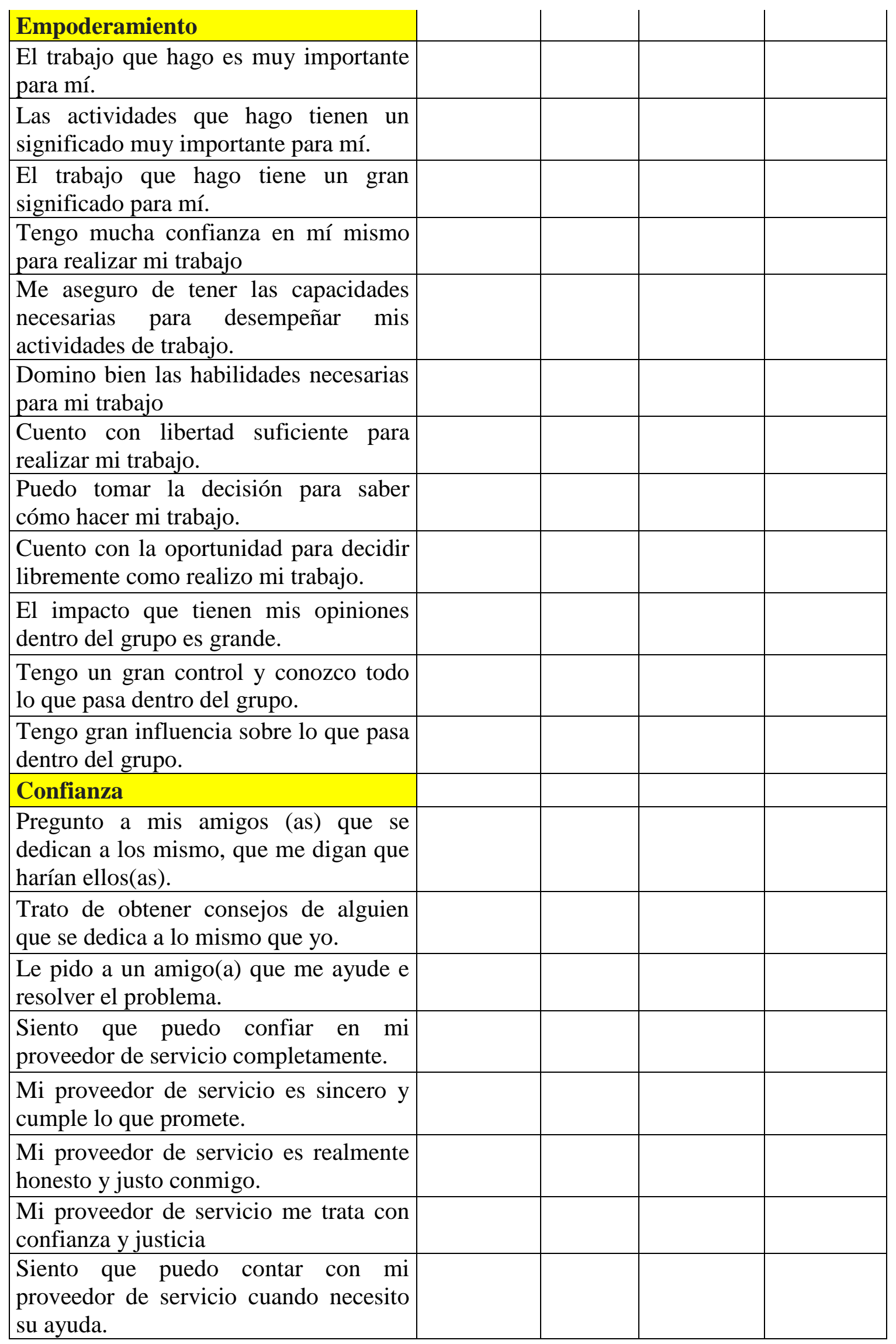




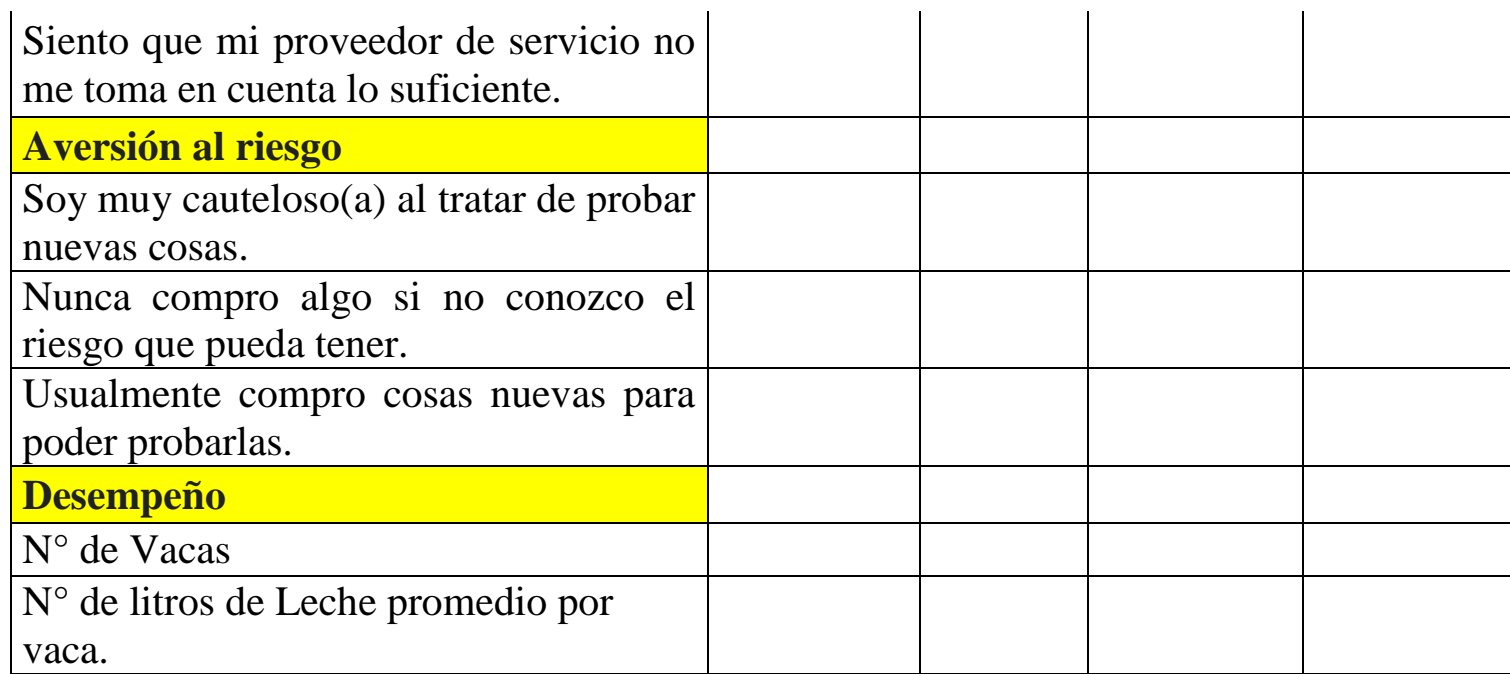

